\title{
SNHGI Promotes Malignant Progression of Glioma by Targeting miR-140-5p and Regulating PI3K/AKT Pathway
}

This article was published in the following Dove Press journal: Cancer Management and Research

\author{
Ren-Duan Cai ${ }^{1}$ \\ Chao-Cai Zhang' \\ Li-Li Xie ${ }^{2}$ \\ Peng-Cheng Wang ${ }^{3}$ \\ Chui-Xue Huang ${ }^{3}$ \\ Jian-Long Chen ${ }^{3}$ \\ Hong-Tao Lv ${ }^{4}$
}

'Department of Neurosurgery, Hainan General Hospital/Hainan Affiliated Hospital of Hainan Medical University, Haikou, Hainan Province, People's Republic of China; ${ }^{2}$ Department of Neurology, Dalian Central Hospital, Dalian, Liaoning Province, People's Republic of China; ${ }^{3}$ Department of Neurosurgery, Hainan People's Hospital, Haikou, Hainan Province, People's Republic of China; ${ }^{4}$ Department of Neurosurgery, The First Affiliated Hospital of Dalian Medical University, Dalian, Liaoning Province, People's Republic of China
Correspondence: Hong-Tao Lv Department of Neurosurgery, The First Affiliated Hospital of Dalian Medical University, No. 222 Zhongshan Road, Xigang District, Dalian II60I I, Liaoning Province, People's Republic of China Tel +86-18098876/25

Email Iht_725@I63.com
Purpose: To explore the regulatory mechanism of long non-coding RNA small nucleolar RNA host gene 1 (SNHG1) in glioma.

Materials and Methods: The expression of SNHG1 and miR-140-5p in glioma tissues and glioma cell lines (LN-18, KNS-81, and KALS-1) was determined, and the effect of the two on cell proliferation, invasion, and PI3K/AKT pathway was analyzed.

Results: SNHG1 was overexpressed in glioma tissues, while miR-140-5p was underexpressed in them, and there was a significant negative correlation between SNHG1 and miR140-5p. In addition, both down-regulation of SNHG1 and up-regulation of miR-140-5p significantly inhibited the malignant proliferation and invasion of glioma, intensified the apoptosis, and also significantly suppressed the activation of the PI3K/AKT pathway. The dual-luciferase reporter assay, RNA pull-down assay, and RIP determination all confirmed that there was a targeting relationship between SNHG1 and miR-140-5p, and there was no difference between KNS-81 and KALS-1 cells transfected with SNHG1+mimics and siSNHG1+inhibitor and those in the si-NC group with unrelated sequences in terms of cell malignant progression.

Conclusion: SNHG1/miR-140-5p axis and its regulation on PI3K/AKT pathway might be a novel therapeutic direction to curb the malignant progression of glioma.

Keywords: $S N H G 1$, glioma, miR-140-5p, PI3K/AKT pathway

\section{Introduction}

Glioma, as a malignant brain tumor, is a common cause of human brain cancerrelated death. ${ }^{1,2}$ According to the epidemiological statistics of glioma, the annual incidence of glioma is approximately 3-8 cases per 100,000 individuals, and the disease is more common in men and people aged 50-69. ${ }^{3,4}$ Glioma is mainly manifested as class II epileptic seizure, and the age of patients is a potential risk factor for the increase of glioma grade. ${ }^{5}$ Patients with glioma are mostly already in the advanced stage at the time of diagnosis, and they are usually treated with surgical resection, radiotherapy, and chemotherapy that is the most common treatment for it. ${ }^{6}$ However, glioma is located in the sensitive central nervous system, so it is difficult for conventional chemotherapy to break through the blood-brain barrier and exert its medicinal properties, which appeals for new treatment strategies. ${ }^{7}$ We would explore the potential therapeutic direction by studying the molecular mechanism of glioma, which is of great value for the treatment of glioma. 
Long non-coding RNA (lncRNA) is a therapeutic direction for glioma. As an endogenous RNA molecule that regulates gene expression at post-transcriptional level, lncRNA is abnormally expressed in cases with one of various diseases including glioma. Dynamic monitoring on it is helpful for us to understand the progress of diseases. ${ }^{8-10}$ For example, urothelial carcinomaassociated 1 (UCA1) can act as a carcinogen for nonsmall cell lung cancer and promotes its development by targeting miR-193a-3p, and dysregulation of growth-arrest -specific transcript 5 (GAS5) is involved in the resistance mechanism of trastuzumab in human epidermal growth factor receptor 2 (HER2) in patients with breast cancer. In addition, IncRNA colon cancer-associated transcript1 (CCAT1) is reported to be able to down-regulate miR$181 \mathrm{~b}$ to promote the malignant progression of glioma. ${ }^{11-13}$ LncRNA is also widely used in drug resistance and clinical diagnosis of glioma. For instance, downregulation of lncRNA PVT1 is beneficial to increasing the anti-drug resistance of paclitaxel in patients with glioma, and IncRNA miR210HG has the potential to be a serum indicator for diagnosis of glioma. ${ }^{14,15}$ Small nucleolar RNA host gene 1 (SNHG1), as a member of the lncRNA family, is an oncogene of glioma. Its up-regulation often indicates malignant progression of patients with glioma. ${ }^{16}$ Studies have revealed that miR-140-5p, as a cancer suppressor, can inhibit the malignant behavior of glioma by partially targeting JAG1. ${ }^{17}$ The SNHG1/miR-140-5p axis mediates the regulatory mechanism of malignant expansion of esophageal cancer cells, but the potential pathological mechanism of the two in glioma has not yet been clarified. ${ }^{18}$ The PI3K/AKT signaling pathway, as one of the classical regulatory pathways of human cancer, is also involved in angiogenesis and cell growth of glioma. ${ }^{19-21}$

We suspected that the regulatory network of SNHG1/ miR-140-5p axis may be involved in the malignant progression of glioma by medicating PI3K/AKT signaling pathway, and we verified it by detecting the expression of SNHG1 and miR-140-5p.

\section{Materials and Methods}

\section{Tissue Sample Collection}

Normal brain tissues and glioma tissues were sampled from patients who had signed informed consent forms. The sampled glioma tissue specimens were assigned to a glioma group $(\mathrm{n}=40)$, including 22 cases in grade I/II and 18 cases in grade III/IV, and sampled normal brain tissue specimens were as assigned to a normal group $(n=30)$. The normal brain tissues were non-tumor brain tissues, which were sampled from patients with craniocerebral injury who had undergone partial resection of brain tissues. Those specimens were sampled from December 2016 to December 2018, and the general data including sex and age of the two groups were comparable $(\mathrm{P}<0.05)$. The experiment was approved by the Ethics Committee of Hainan People's Hospital, and operations and tissue storing were carried out in accordance with corresponding standards.

\section{Cell Culturing}

Human glioma cell lines (LN-18, KNS-81, and KALS-1) and normal glial cells (HEB) (C0786, C1648, GDC0098632A65190, and DA-C5504, Guandao Biological Engineering Co., Ltd., Shanghai, China) were cultured in dulbecco's modified eagle (DMEM) (BH-S3208, Bohu Biotechnology Co., Ltd., Shanghai, China) supplemented with $10 \%$ phosphate buffer saline (PBS, 120882, Chreagen Biotechnology Co., Ltd., Beijing, China), $100 \mathrm{U} / \mathrm{mL}$ penicillin (07500, Yihui Biological Technology Co. Ltd., Shanghai, China), and $100 \mathrm{mg} / \mathrm{mL}$ streptomycin (YSH106-01, Yanjin Biological Co., Ltd., Shanghai, China) under $5 \% \mathrm{CO}_{2}$ at $37^{\circ} \mathrm{C}$.

\section{Cell Transfection}

MiR-140-5p overexpression sequence (mimics), miR-140$5 \mathrm{p}$ inhibition sequence (inhibitor), miR negative control (miR-NC), targetedly inhibited SNHG1 RNA (si-SNHG1), targetedly overexpressed SNHG1 RNA (SNHG1), and negative control RNA (si-NC) were transfected into human glioma cells by a Lipofectamine ${ }^{\mathrm{TM}} 2000$ Kit (11668, Biomics Biotechnologies Co., Ltd., Nantong, China) in strict accordance with the kit instructions.

\section{qRT-PCR Assay}

Total RNA was extracted from collected glioma tissues and cells with a TRIzol Kit (KL058, Kanglang Biotechnology Co., Ltd., Shanghai, China), and cDNA was synthesized using a Bio-Rad Ssofast EvaGreen Supermix Kit (1725202, Yihui Biological Technology Co. Ltd., Shanghai, China) and Stepone Plus fluorescence ration PCR instrument (Biocytocare Biotechnology Co., Ltd., Beijing, China). All primers were designed and synthesized by Shanghai Qiantu Biotechnology Co., Ltd. U6 and $\beta$-Actin were taken as internal references for miRNA and mRNA, respectively, and data of this experiment were analyzed using $2-\Delta \Delta$ ct. 


\section{Western Blotting Assay}

Total RNA was extracted from cells in each group that were cultured in RIPA lysate (PS0033, Zhenyu Biotechnology Co., Ltd., Shanghai, China), and its concentration was detected using a BCA Kit (LCB004, Junrui Biotechnology Co., Ltd., Shanghai, China), and adjusted to $4 \mu \mathrm{g} / \mu \mathrm{L}$. Subsequently, the RNA was separated through $12 \%$ sodium dodecyl sulfate-polyacrylamide gel electrophoresis (SDS-PAGE) (LM0053A, LMAI Bioengineering Co., Ltd., Shanghai, China), and then transferred to a polyvinylidene fluoride (PVDF) membrane (ISEQ00011, Chreagen Biotechnology Co., Ltd. Beijing, China). The membrane was sealed with $5 \%$ skim milk $(\mathrm{N} /$ A-433, Lianshuo Biological Technology Co., Ltd., Shanghai, China) for $4 \mathrm{~h}$, and then added with AMPK, p-AMPK, PI3K, and p-AKT (1:1000) and $\beta$-Actin primary antibody $(1: 1000)$, and sealed at $4^{\circ} \mathrm{C}$ overnight. The membrane was washed to remove the primary antibody, and then it was added with horseradish peroxidase-labeled goat anti-rabbit secondary antibody (1:2000), and cultured at $37^{\circ} \mathrm{C}$ for $1 \mathrm{~h}$. Antibodies were all purchased from Beijing Future Biotechnology Co., Ltd. The chemiluminescence was detected using an electrochemiluminescence (ECL) kit (KL-16664, Kalang Biotechnology Co., Ltd., Shanghai, China) and Bio-Rad ChemiDoc MP Imaging System (17001402, Yihui Biological Technology Co. Ltd., Shanghai, China), and the grey value was analyzed using Quantity One.

\section{Cell Proliferation Assay}

The proliferation of glioma cells was determined using a MTT Kit (EY-19002, Yiyan Biotechnology Co., Ltd., Shanghai, China). The cells were seeded into 96-well plates at $5 \times 10^{3}$ cells/well, and then the plates were added with $20 \mu \mathrm{L} 5 \mu \mathrm{mg} / \mathrm{mL}$ MTT solution, and cultured at $37^{\circ} \mathrm{C}$ for $24,48,72$ and $96 \mathrm{~h}$, separately. The plates were added with $200 \mu \mathrm{L}$ dimethyl sulfoxide at each culturing time point, and then the optical density (OD) of cells in each group at $450 \mathrm{~nm}$ absorbance was determined using a V-1200 spectrophotometer (Hengfei Biotechnology Co., Ltd., Shanghai, China).

\section{Cell Apoptosis Assay}

The transfected cells were digested by $0.25 \%$ trypsin (T4049-500ML, Bei Nuo Biotechnology Co., Ltd., Shanghai, China). After digestion, the cells were washed with PBS twice, added with $100 \mu \mathrm{L}$ binding buffer to prepare $1 \times 10^{6}$ cells $/ \mathrm{mL}$ suspension. The suspension was added with AnnexinV-FITC and PI in order, incubated at room temperature in the dark for $5 \mathrm{~min}$, and finally detected using a flow cytometer (AMG0002051, Image Trading Co., Ltd., Beijing, China). The experiment was repeated three times, and the results were averaged.

\section{Cell Invasion Assay}

Glioma cells transfected for $24 \mathrm{~h}$ were collected and seeded into 24 -well plates at $3 \times 10^{4}$ cells/well. The cells were digested with trypsin, and transferred to the upper compartment. The upper compartment was added with $200 \mu \mathrm{L}$ RPMI 1640 culturing solution, and the lower compartment was added with 500mL RPMI 1640 supplemented with $10 \%$ FBS. The plate was incubated at $37^{\circ} \mathrm{C}$ for $48 \mathrm{~h}$. The substrate and cells that did not penetrate the membrane surface in the upper compartment were wiped off. The plates were washed with PBS three times, immobilized with paraformaldehyde for $10 \mathrm{~min}$, and then washed with double-distilled water three times. Finally, the plates were stained with $0.5 \%$ crystal violet after being dried naturally. The invasion of the cells was evaluated using a microscope.

\section{Dual-Luciferase Reporter (DLR) Assay}

The fragment for predicting binding locus of miR-140-5p from SNHG1 was cloned into the pmirG10 dual-luciferase miRNA target expression vector (Promega) to generate the report vector SNHG1-wild type (SNHG1-Wt). With the aim of mutating the putative binding loci of miR-140-5p in SNHG1, the sequence of the putative binding loci was replaced as indicated and named SNHG1 mutant (SNHG1Mut).

\section{RNA Pull-Down Assay and RNA-Binding Protein Immunoprecipitation (RIP) Assay} A RNeasy Mini Kit (DXT-74126, Kemin Biotechnology Co., Ltd., Shanghai, China) was adopted to purify biotinylated SNHG1. Then, $3 \mu \mathrm{g}$ purified SNHG1 was incubated with $1 \mathrm{mg} \mathrm{KNS}-81$ or KALS- 1 cell lysate at $25^{\circ} \mathrm{C}$ for $1 \mathrm{~h}$, and then recovered by streptavidin-agarose beads. Finally, RNA in the pull-down materials was quantitatively determined by qRT-PCR.

A Magna RIP reagent kit (17-700, Fushen Biotechnology Co, Ltd., Shanghai, China) was adopted for RIP assay. The properties of antibody beads, including connection and configuration, were evaluated. The thawed 
antibody beads were suspended and mixed with samples, and incubated at $4^{\circ} \mathrm{C}$ overnight. The suspension was placed on a magnetic frame and washed with buffer solution. After immunoprecipitation, the co-immunoprecipitated products were harvested. RNA was extracted and purified, and the abundance of target RNA was determined.

\section{Statistical Analyses}

In this study, the collected data were analyzed statistically and visualized into required figures using GraphPad 6. Inter-group comparison was carried out by the independent $t$ test, and multi-group comparison was carried out by the one-way ANOVA, expressed by F. Post hoc pairwise comparison was carried out using the LSD- $t$ test, and comparison in expression at different time points was carried out using the repeated measures analysis of variance, and expressed by F, and Bonferroni post hoc test was adopted. In addition, Pearson's correlation analysis was carried out. $\mathrm{P}<0.05$ indicates a significant difference.

\section{Results}

\section{Clinical Value of SNHGI and miR-I40-5p in Glioma}

Glioma tissues showed significantly higher SNHG1 expression and significantly lower miR-140-5p expression than normal brain tissues (both $P<0.05$ ). Further analysis of the correlation between SNHG1 and miR-140-5p showed that SNHG1 was significantly negatively correlated with miR-140-5p $(\mathrm{r}=-0.773, \mathrm{P}<0.001)$. Figure 1 .

\section{Effect of SNHGI on the Malignant Progression of Glioma Cells}

Significantly higher SNHG1 expression was found in KNS81 and KALS- 1 cells, so KNS- 81 and KALS- 1 cells were selected for transfection analysis. It came out that KNS-81 and KALS-1 cells transfected with si-SNHG1 showed decreased SNHG1 expression and those transfected with SNHG1 showed increased SNHG1 expression. Cell behavior studies revealed that compared with KNS-81 and KALS-1 cells transfected with si-NC, those transfected with SNHG1 showed significantly increased proliferation and invasion and significantly decreased apoptosis, while compared with KNS-81 and KALS-1 cells transfected with si-NC, those transfected with si-SNHG1 showed significantly decreased proliferation and invasion and significantly increased apoptosis (all $\mathrm{P}<0.05$ ). Figure 2 .

\section{Effect of miR-140-5p on the Malignant Progression of Glioma Cells}

Significantly lower miR-140-5p expression was found in KNS- 81 and KALS- 1 cells, so KNS- 81 and KALS- 1 were selected for transfection analysis. It came out that KNS-81 and KALS-1 cells transfected with mimics showed increased miR-140-5p expression and those transfected with inhibitor showed decreased miR-140-5p expression. Cell behavior studies revealed that compared with KNS-81 and KALS-1 cells transfected with miR-NC, those transfected with inhibitor showed significantly increased proliferation and invasion and significantly decreased apoptosis, while compared with KNS-81 and KALS- 1 cells transfected with miR-NC, those transfected with mimics showed significantly decreased proliferation and invasion and significantly increased apoptosis $(\mathrm{P}<0.05)$. Figure 3 .

\section{Effects of SNHGI and miR-I40-5p on the PI3K/AKT Pathway}

Cells transfected with SNHG1 or inhibitor showed significantly up-regulated AMPK, p-AMPK, PI3K, and p-AKT,
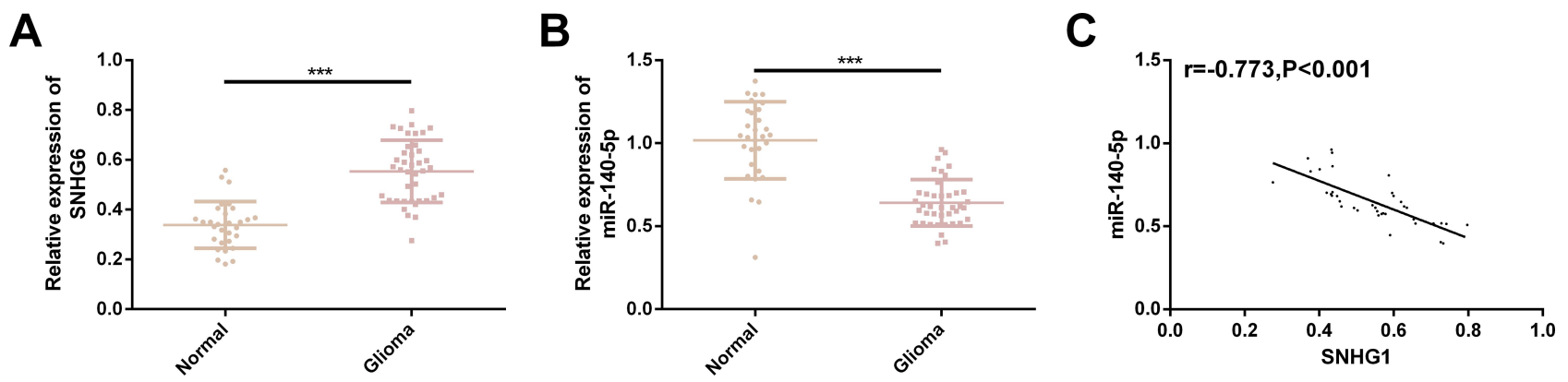

Figure I Clinical value of SNHGI and miR-140-5p in glioma. (A) SNHGI was significantly highly expressed in glioma tissues. (B) MiR-I40-5p was significantly lowly expressed in glioma tissues. (C) SNHGI was significantly negatively correlated with miR-140-5p $(r=-0.773, P<0.001)$.

Note: ****ndicates $\mathrm{P}<0.001$.

Abbreviations: SNHGI, small nucleolar RNA host gene I; miR, microRNA. 


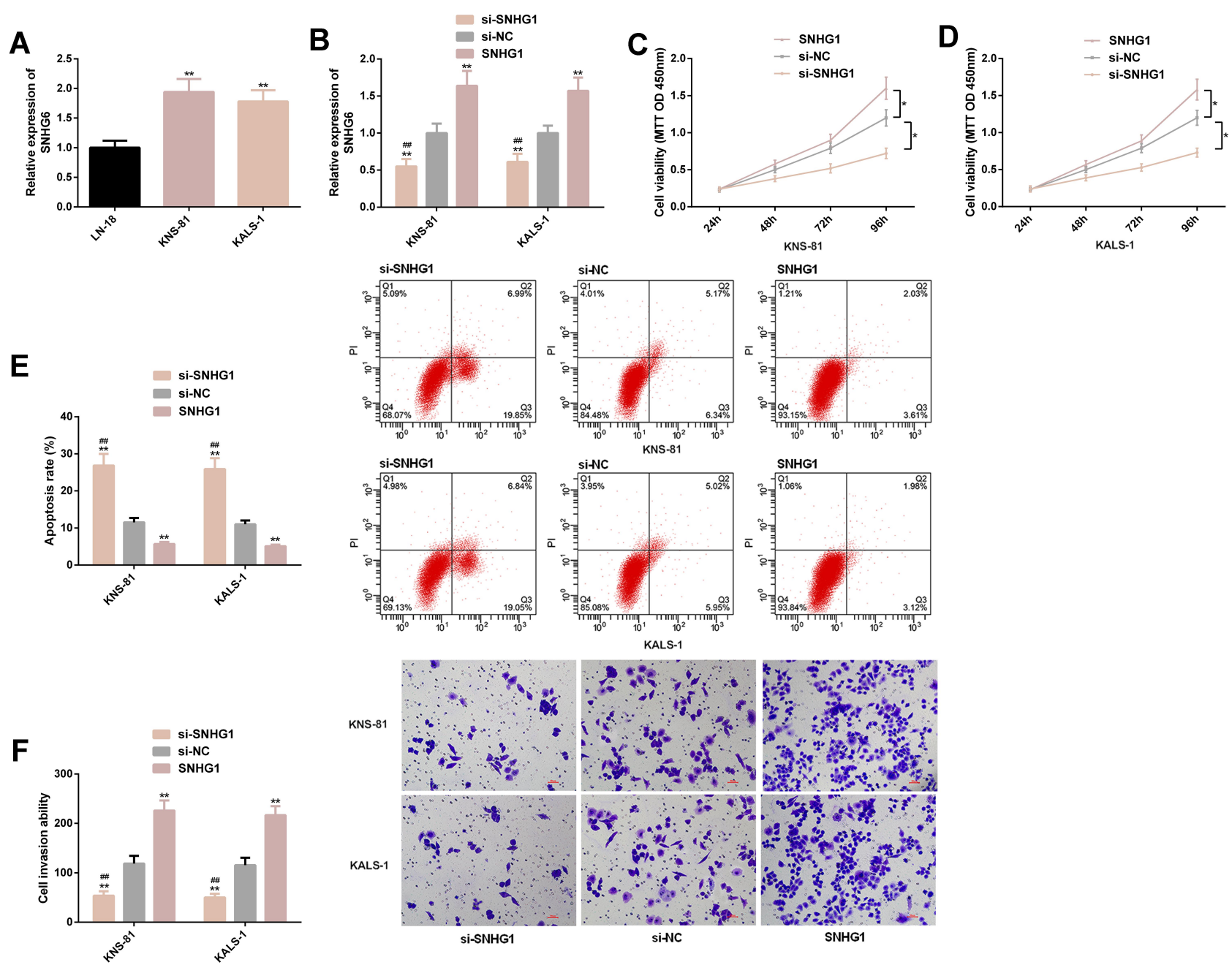

Figure 2 Effects of SNHGI on malignant progression of glioma cells. (A) KNS-8I and KALS-I cells showed higher SNHGI expression. (B) Expression of SNHGI in KNS-8 I and KALS-I after transfection. (C) Proliferation of KNS-8I cells. (D) Proliferation of KALS-I cells. (E) Apoptosis rate of KNS-8I and KALS-I cells, and its flow cytometry profiling. (F) Invasion of KNS-8I and KALS-I cells, and its microscopic photographs.

Notes: *Indicates $\mathrm{P}<0.05$ vs $\mathrm{LN}-18$ cells or the si-NC group; ${ }^{* *}$ Indicates $\mathrm{P}<0.0 \mathrm{I}$ vs $\mathrm{LN}-18$ cells or the si-NC group; ${ }^{\# \#}$ Indicates $\mathrm{P}<0.0 \mathrm{I}$ vs the $\mathrm{SNHGI}$ group.

Abbreviations: SNHGI, small nucleolar RNA host gene I; NC, negative control; si, short interfering; MTT, 3-(4,5)-dimethylthiahiazo (-z-yl)-3,5-diphenytetrazoliumromide; OD, optical density; PI, propidium iodide.

while those transfected with si-SNHG1 or mimics showed a significant down-regulation of them. Figure 4.

\section{Identification of Target Genes of SNHG I}

We found through starBase website that there were targeting binding locus between SNHG1 and miR-140-5p, and the DLR assay revealed that up-regulation of miR-140-5p significantly lowered the luciferase activity of SNHG13'UTR-Wt $(\mathrm{P}<0.05)$, but exerted no effect on that of SNHG13'UTR-Mut $(\mathrm{P}>0.05)$. In the RNA pull-down assay, miR-140-5p was pulled down only by SNHG13'UTR-Wt $(\mathrm{P}<0.05)$, but not affected by SNHG13'UTR-Mut ( $>>0.05)$. RIP analysis showed that under the action of miR-140-5p mimics, SNHG1 in KNS81 and KALS- 1 cells was significantly up-regulated $(\mathrm{P}<$
0.05). In addition, real-time PCR detection revealed that KNS-81 and KALS-1 cells transfected with si-SNHG1 showed significantly increased miR-140-5p expression $(\mathrm{P}<0.05)$. Figure 5.

\section{Co-Transfection Experiment}

KNS-81 and KALS-1 cells transfected with SNHG1 + mimics or si-SNHG1+inhibitor were not different from those transfected with si-NC in cell invasion, proliferation, and apoptosis, and the PI3K/AKT pathway-related protein levels (all $\mathrm{P}>0.05$ ), but showed significantly stronger proliferation and invasion, significantly weaker apoptosis, and significantly higher AMPK, p-AMPK, PI3K, and p-AKT protein levels than those transfected with si-SNHG1, and also showed significantly weaker proliferation and 

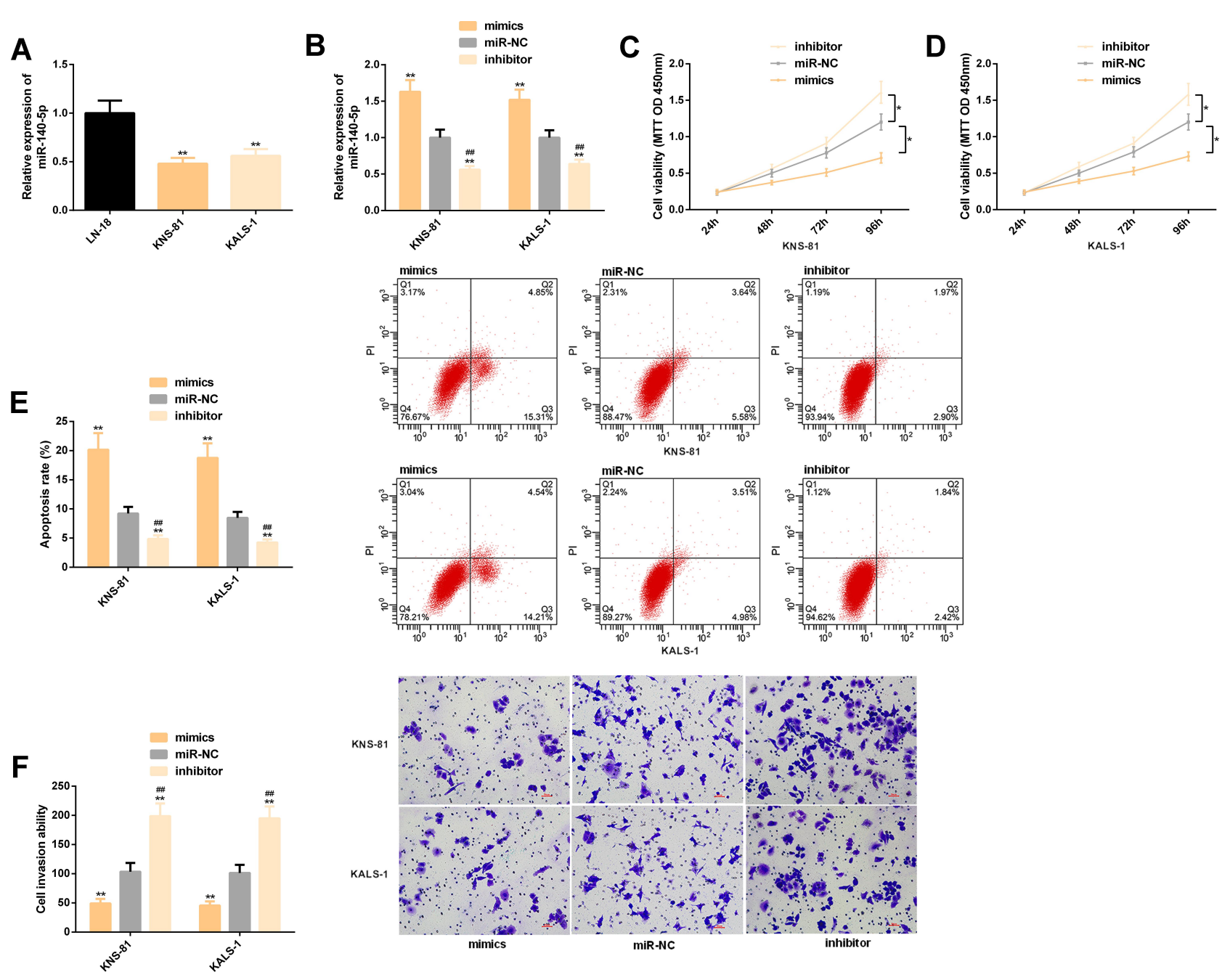

Figure 3 Effects of miR-I40-5p on malignant progression of glioma cells. (A) KNS-8I and KALS-I cells showed lower miR-I40-5p expression. (B) The expression of miR140-5p in KNS-8I and KALS-I cells after transfection. (C) Proliferation of KNS-8I cells. (D) Proliferation of KALS-I cells. (E), Apoptosis rate of KNS-8I and KALS-I cells, and its flow cytometry profiling. (F) Invasion of KNS-8I and KALS-I cells, and its microscopic photographs.

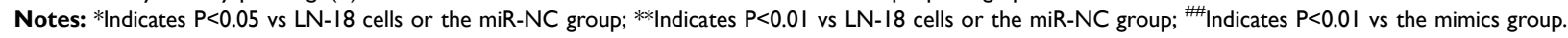

Abbreviations: miR, microRNA; NC, negative control; MTT, 3-(4,5)-dimethylthiahiazo (-z-yl)-3,5-di- phenytetrazoliumromide; OD, optical density; PI, propidium iodide.

invasion, significantly stronger apoptosis, and significantly lower levels of proteins than those transfected with SNHG1 (all $\mathrm{P}<0.05$ ). Figure 6.

\section{Discussion}

SNHG1 is a regulatory factor with dynamic expression changes in various cancers, and is widely used for diseases including the above-mentioned glioma, prostate cancer, hepatic carcinoma, and colon cancer. ${ }^{22,23}$ An increasing number of researchers show enthusiasm for studying the role of SNHG1 in glioma, and many research results appear one after another. For example, one study by $\mathrm{Li}$ et $\mathrm{al}^{24}$ has pointed out that SNHG1 can regulate the miR154-5p/miR-376b-3p-FOXP2-KDM5B network to affect the behavior of glioma cells. One other study by Liu et $\mathrm{al}^{25}$ has reported that SNHG1 is overexpressed in glioma tissues and cell lines, and it is involved in the pathological progress of glioma through negative regulation on miR194 and PHLDA1. Moreover, there are many reports about the regulatory effects of miR-140-5p on glioma. MiR-140$5 \mathrm{p}$ can not only targetedly inhibit ADAM9 and further regulate glioma growth and metastasis but also accept the regulation by IncRNA HOXA11 to affect the cell proliferation, cycle and apoptosis. ${ }^{26,27}$

In this study, both SNHG1 and miR-140-5p showed abnormal expression in glioma tissues. The expression of SNHG1 was relatively high, while that of miR-140-5p was opposite, implying that they might participate in the progression of glioma. The analysis on the correlation between SNHG1 and miR-140-5p showed that SNHG1 was 

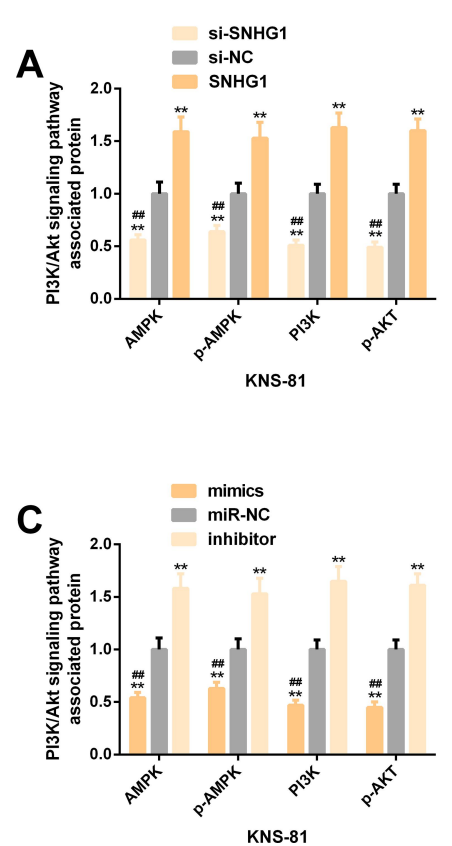
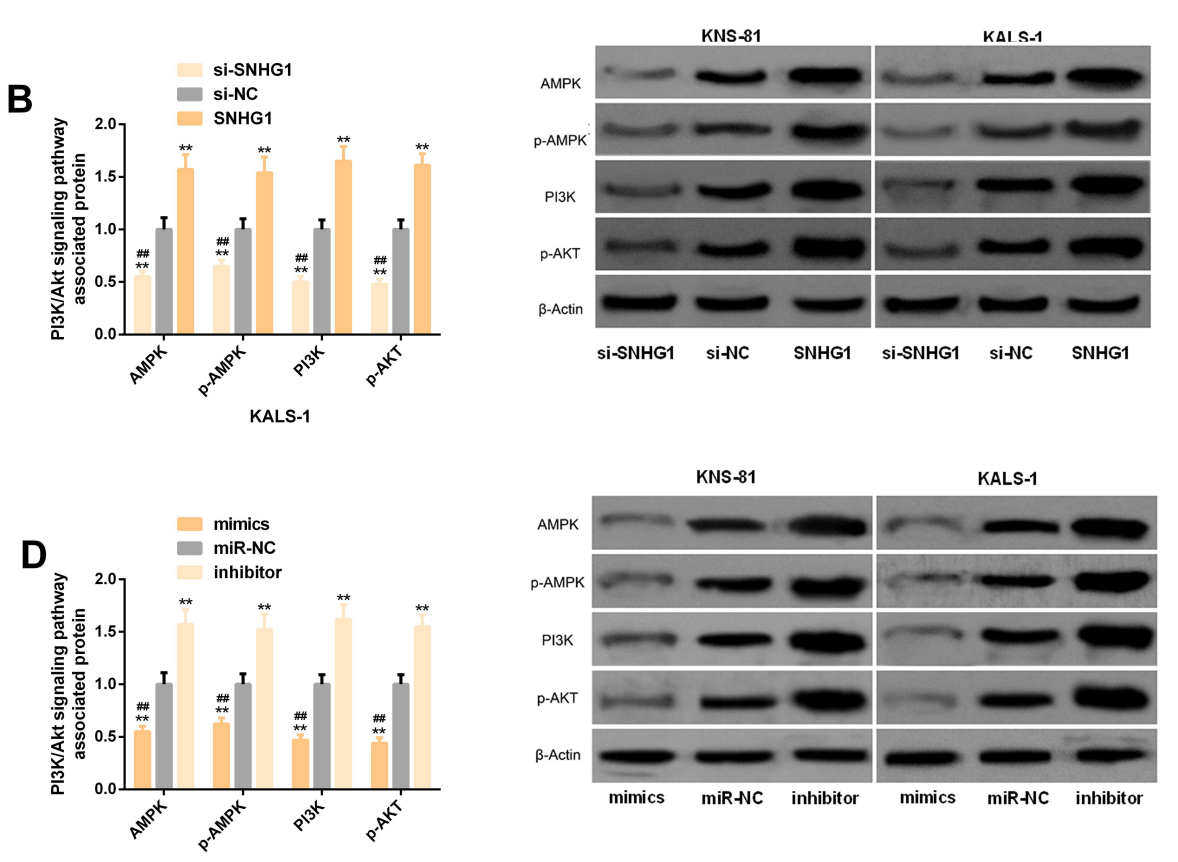

Figure 4 Effects of SNHGI and miR-I40-5p on the PI3K/AKT pathway. (A) Effects of SNHGI on the PI3K/AKT pathway-related protein levels in transfected KNS-8I cells. (B) Effects of SNHGI on the PI3K/AKT pathway-related protein levels in transfected KALS-I cells, and its correspond protein profiling. (C) Effects of miR-I40-5p on the PI3K/AKT pathway-related protein levels in transfected KNS-8I cells. (D) Effects of miR-140-5p on the PI3K/AKT pathway-related protein levels in transfected KALS-I cells, and its corresponding protein profiling.

Notes: **Indicates $\mathrm{P}<0.01$ vs the si-NC group; ${ }^{\#}$ Indicates $\mathrm{P}<0.01$ vs the SNHGI group.

Abbreviations: SNHGI, small nucleolar RNA host gene I; miR, microRNA; NC, negative control; si, short interfering; PI3K, phosphatidylinositol 3 kinase; AKT, protein kinase B; P-AKT, phosphorylated-protein kinase B; AMPK, AMP-activated protein kinase; P-AMPK, phosphorylated-AMP-activated protein kinase.

significantly negatively correlated with miR-140-5p, suggesting that the two may play antagonistic roles in the pathological process of glioma. The subsequent in vitro experiments revealed that the expression of SNHG1 and miR-140-5p in glioma cell lines was consistent with that of them in tissue samples, and was also consistent with the previous research results. We selected two glioma cell lines (KNS-81 and KALS-1) with the most significant differential expression to carry out transfection research, and upregulated and down-regulated SNHG1 and miR-140-5p in them, respectively. It came out that transfection of shSNHG1 or inhibitor strongly intensified the proliferation and invasion of KNS-81 and KALS-1 cells, and strongly weakened the apoptosis of them, while transfection of siSNHG1 or mimics exerted opposite effects on the cells. It indicates that down-regulating SNHG1 or up-regulating miR-140-5p might become potential therapeutic targets for glioma, and help to inhibit malignant progression of it. Previous studies have shown that the PI3K/AKT pathway plays a part in the regulation mechanism of invasion, proliferation, and apoptosis of human glioma cells. AMPK, p-AMPK, PI3K and p-AKT are important PI3K/AKT pathway-related factors, of which AMPK and p-AMPK are upstream regulators of the PI3K/AKT pathway, and inhibition on related proteins of this pathway can exert anti-tumor activity. ${ }^{28}$ Wei et $\mathrm{al}^{29}$ have reported that activating the PI3K/AKT pathway promotes the malignant progression of glioma and stimulates the tumorigenic ability of glioma stem cells, which is one of the mechanisms of action. Therefore, we also investigated the regulation of SNHG1 and miR-140-5p on the PI3K/AKT pathway. It came out that both down-regulation of SNHG1 and up-regulation of miR-140-5p could effectively inhibit the activation of the $\mathrm{PI} 3 \mathrm{~K} / \mathrm{AKT}$ pathway, which was manifested as significantly down-regulation of PI3K/AKT pathway-related proteins such as PI3K, p-AKT, AMPK, and p-AMPK. The results suggest that SNHG1 and miR-140-5p have important regulatory effects on the activation of the PI3K/AKT pathway.

On the other hand, we verified the targeted relationship between SNHG1 and miR-140-5p through a DLR assay. The results revealed that knock-down of SNHG1 strongly lowered the luciferase activity of miR-140-5p3'UTR-Wt, but exerted no effect on that of miR-140-5p3'UTR-Mut, and transfection of si-SNHG1 significantly increased the expression of miR-140-5p. In addition, in the RNA pull-down assay, only SNHG13'UTR-Wt obviously pulled down miR- 
A

SNHG1

5'...AGACCUUGUAG-AUAACCACUC...3'

miR-140-5p

3'...GAUGGUAUCCCAUUUUGGUGAC...5'

B

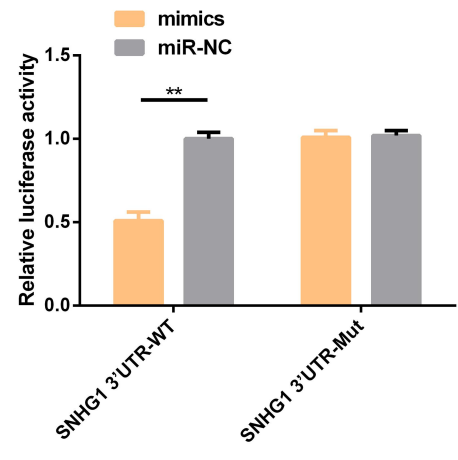

KNS-81

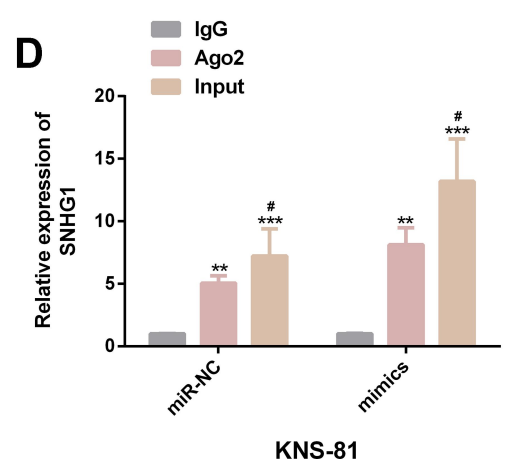

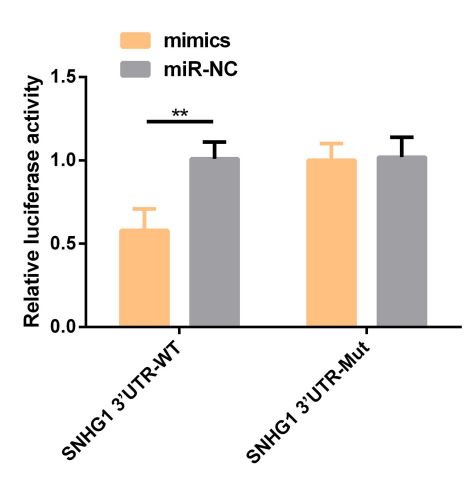

KALS-1

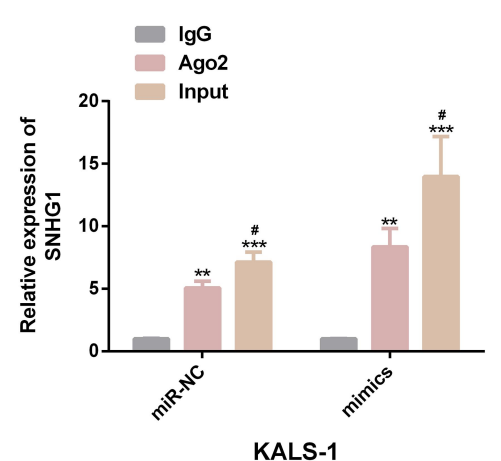

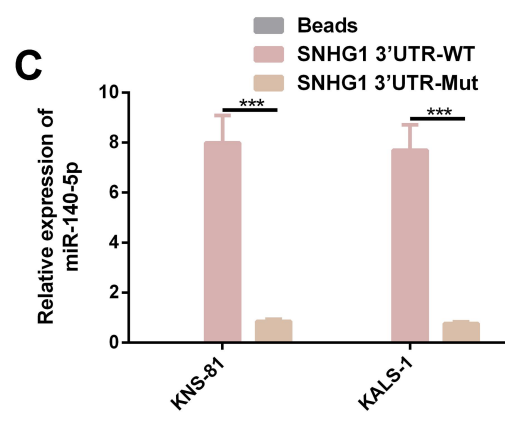

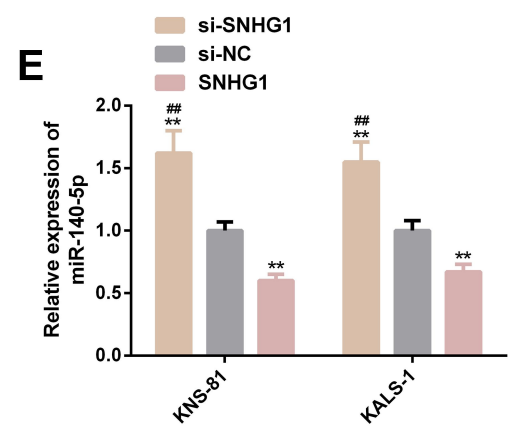

Figure 5 Identification of target genes of SNHGI. (A) There were targeting binding locus between SNHGI and miR-I40-5p. (B) Relative luciferase activity - DLR assay. (C) RNA pull-down assay. (D) RNA analysis. (E) Expression of miR-I40-5p in transfected KNS-8I and KALS-I cells.

Notes: **Indicates that in terms of inter-group comparison or in comparison with the si-NC group, $\mathrm{P}<0.0 \mathrm{O}$; ***Indicates that in terms of inter-group comparison or in

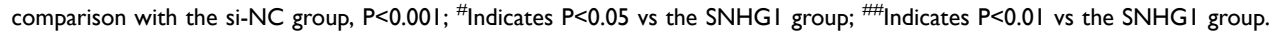

Abbreviations: SNHGI, small nucleolar RNA host gene I; miR, microRNA; NC, negative control; DLR, dual-luciferase reporter; WT, wild type; Mut, mutant; IgG, immunoglobulin G; Ago2, Argonaute2.

140-5p. RIP analysis showed that under the action of miR140-5p mimics, SNHG1 in KNS-81 and KALS-1 cells was significantly up-regulated. The above verification experiments of gene relationship all indicate that SNHG1 can specifically bind to miR-140-5p, and has negative regulation on the expression of miR-140-5p. We also performed cotransfection experiments, and found that KNS-81 and KALS-1 cells transfected with SNHG1+mimic and siSNHG1+inhibitor were not different from those in the si$\mathrm{NC}$ group with unrelated sequences in cell malignant progression, but showed significantly stronger proliferation and invasion, significantly weaker apoptosis, and significantly higher levels of PI3K/AKT-related proteins than those transfected with si-SNHG1, and also showed significantly weaker proliferation and invasion, significantly stronger apoptosis, and significantly lower levels of proteins than those transfected with sh-SNHG1. Those results once again confirmed the existence of a targeted regulatory relationship between SNHG1 and miR-140-5p. The above results implied that SNHG1 could promote the malignant progression of glioma by targeting miR-140-5p and activating the PI3K/ AKT pathway, and down-regulating SNHG1 or upregulating miR-140-5p may be potential targets for inhibiting malignant progression of glioma.

\section{Conclusion}

The regulatory network of the SNHG1/miR-140-5p axis is a novel potential therapeutic direction to curb the malignant progression of glioma. However, there is still a room for improvement in this study. First of all, we can supplement the research on potential drug resistance of SNHG1 and miR-140-5p in glioma. Secondly, we can supplement 

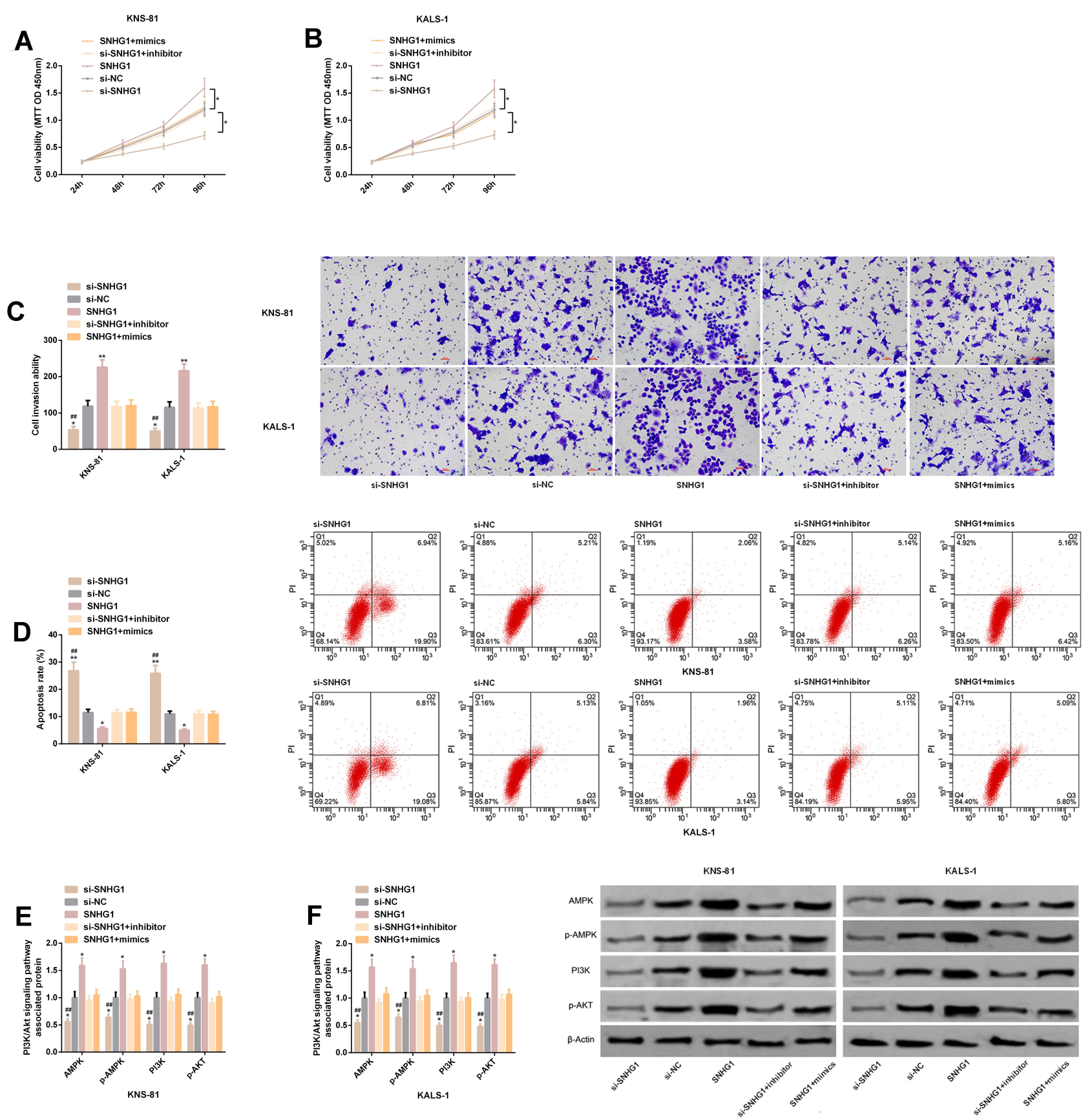

Figure 6 Co-transfection experiment. (A) Proliferation of KNS-8I cells transfected with SNHGI+mimics. (B) Proliferation of KALS-I cells transfected with SNHGI +mimics. (C) Invasiveness of KNS-8I and KALS-I cells transfected with SNHGI+mimics, and its microscopic photographs. (D) Apoptosis of KNS-8I and KALS-I cells transfected with SNHGI+mimics and flow cytometry. (E-F) Levels of PI3K/AKT pathway-related proteins in KNS-8I and KALS-I cells transfected with SNHGI+mimics, and their protein profiling.

Notes: *Indicates $\mathrm{P}<0.05$ vs the si-NC group; **Indicates $\mathrm{P}<0.0 \mathrm{I}$ vs the si-NC group; ${ }^{\#}$ Indicates $\mathrm{P}<0.0 \mathrm{I}$ vs the SNHGI group.

Abbreviations: SNHGI, small nucleolar RNA host gene I; miR, microRNA; NC, negative control; si, short interfering; MTT, 3-(4,5)-dimethylthiahiazo (-z-yl)-3,5-diphenytetrazoliumromide; OD, optical density; PI, propidium iodide; PI3K, phosphatidylinositol 3 kinase; AKT, protein kinase B; p-AKT, phosphorylated-protein kinase B; AMPK, AMP-activated protein kinase; P-AMPK, phosphorylated-AMP-activated protein kinase.

an analysis on the value of both in the diagnosis of pathological parameters of patients. In addition, we can explore the downstream target mRNA of miR-140-5p.

\section{Disclosure}

The authors report no conflicts of interest in this work.

\section{References}

1. Miller AM, Shah RH, Pentsova EI, et al. Tracking tumour evolution in glioma through liquid biopsies of cerebrospinal fluid. Nature. 2019;565(7741):654-658.

2. Ludwig K, Kornblum HI. Molecular markers in glioma. J Neurooncol. 2017;134(3):505-512. doi:10.1007/s11060-017-2379-y 
3. Zhang N, Shuai K, Cheng J, Yang W, Kan Z. LncRNA linc01116 prometes glioma cell migration and invasion by modulation of radixin targeted by miR-31. Int $J$ Clin Exp Pathol. 2019;12 (3):1078-1086.

4. Amirian ES, Armstrong GN, Zhou R, et al. The glioma international case-control study: a report from the genetic epidemiology of glioma international consortium. Am J Epidemiol. 2016;183(2):85-91.

5. Rasmussen BK, Hansen S, Laursen RJ, et al. Epidemiology of glioma: clinical characteristics, symptoms, and predictors of glioma patients grade I-IV in the the Danish Neuro-Oncology Registry. $J$ Neurooncol. 2017;135(3):571-579. doi:10.1007/s11060-017-2607-5

6. Ma CC, Xiong Z, Zhu GN, et al. Long non-coding RNA ATB promotes glioma malignancy by negatively regulating miR-200a. $J$ Exp Clin Cancer Res. 2016;35(1):90. doi:10.1186/s13046-016-0367-2

7. Kundu M, Das S, Dhara D, Mandal M. Prospect of natural products in glioma: a novel avenue in glioma management. Phytother Res. 2019;33(10):2571-2584. doi:10.1002/ptr.6426

8. Reon BJ, Anaya J, Zhang Y, et al. Expression of lncRNAs in low-grade gliomas and glioblastoma multiforme: an in silico analysis. PLoS Med. 2016;13(12):e1002192. doi:10.1371/journal. pmed.1002192

9. Hao NB, He YF, Li XQ, Wang K, Wang RL. The role of miRNA and lncRNA in gastric cancer. Oncotarget. 2017;8(46):81572-81582. doi:10.18632/oncotarget.19197

10. Wang W, Zhao Z, Yang F, et al. An immune-related lncRNA signature for patients with anaplastic gliomas. J Neurooncol. 2018;136 (2):263-271. doi:10.1007/s11060-017-2667-6

11. Nie W, Ge HJ, Yang XQ, et al. LncRNA-UCA1 exerts oncogenic functions in non-small cell lung cancer by targeting miR-193a-3p. Cancer Lett. 2016;371(1):99-106. doi:10.1016/j.canlet.2015.11.024

12. Li W, Zhai L, Wang H, et al. Downregulation of LncRNA GAS5 causes trastuzumab resistance in breast cancer. Oncotarget. 2016;7 (19):27778-27786. doi:10.18632/oncotarget.8413

13. Cui B, Li B, Liu Q, Cui Y. IncRNA CCAT1 promotes glioma tumorigenesis by sponging miR-181b. J Cell Biochem. 2017;118 (12):4548-4557. doi:10.1002/jcb.26116

14. Song T, Yan L, Cai K, Zhao T, Xu M. Downregulation of long noncoding RNA PVT1 attenuates paclitaxel resistance in glioma cells. Cancer Biomark. 2018;23(3):447-453. doi:10.3233/CBM-181573

15. Min W, Dai D, Wang J, et al. Long noncoding RNA miR210HG as a potential biomarker for the diagnosis of glioma. PLoS One. 2016;11 (9):e0160451. doi:10.1371/journal.pone.0160451

16. Wang Q, Li Q, Zhou P, et al. Upregulation of the long non-coding RNA SNHG1 predicts poor prognosis, promotes cell proliferation and invasion, and reduces apoptosis in glioma. Biomed Pharmacother. 2017;91:906-911. doi:10.1016/j.biopha.2017.05.014

17. Yang HL, Gao YM, Zhao JA. miR1405p inhibits human glioma cell growth and invasion by targeting JAG1. Mol Med Rep. 2017;16 (3):3634-3640. doi:10.3892/mmr.2017.6951
18. Zhang K, Chen J, Song H, Chen LB. SNHG16/miR-140-5p axis promotes esophagus cancer cell proliferation, migration and EMT formation through regulating ZEB1. Oncotarget. 2018;9(1):102 8-1040. doi:10.18632/oncotarget.23178

19. Mayer IA, Arteaga CL. The PI3K/AKT pathway as a target for cancer treatment. Annu Rev Med. 2016;67:11-28. doi:10.1146/ annurev-med-062913-051343

20. Zhu Y, Zhang X, Qi L, et al. HULC long noncoding RNA silencing suppresses angiogenesis by regulating ESM-1 via the PI3K/Akt/ mTOR signaling pathway in human gliomas. Oncotarget. 2016;7 (12):14429-14440. doi:10.18632/oncotarget.7418

21. Zhang L, Liang X, Li Y. Long non-coding RNA MEG3 inhibits cell growth of gliomas by targeting miR-93 and inactivating PI3K/AKT pathway. Oncol Rep. 2017;38(4):2408-2416. doi:10.3892/or.2017. 5871

22. Li J, Zhang Z, Xiong L, et al. SNHG1 lncRNA negatively regulates miR-199a-3p to enhance CDK7 expression and promote cell proliferation in prostate cancer. Biochem Biophys Res Commun. 2017;487 (1):146-152. doi:10.1016/j.bbrc.2017.03.169

23. Zhang M, Wang $\mathrm{W}$, Li $\mathrm{T}$, et al. Long noncoding RNA SNHG1 predicts a poor prognosis and promotes hepatocellular carcinoma tumorigenesis. Biomed Pharmacother. 2016;80:73-79. doi:10.1016/ j.biopha.2016.02.036

24. Li H, Xue Y, Ma J, et al. SNHG1 promotes malignant biological behaviors of glioma cells via microRNA-154-5p/miR-376b-3pFOXP2- KDM5B participating positive feedback loop. J Exp Clin Cancer Res. 2019;38(1):59. doi:10.1186/s13046-019-1063-9

25. Liu L, Shi Y, Shi J, et al. The long non-coding RNA SNHG1 promotes glioma progression by competitively binding to miR-194 to regulate PHLDA1 expression. Cell Death Dis. 2019;10(6):463. doi:10.1038/s41419-019-1698-7

26. Liu X, Wang S, Yuan A, Yuan X, Liu B. MicroRNA-140 represses glioma growth and metastasis by directly targeting ADAM9. Oncol Rep. 2016;36(4):2329-2338. doi:10.3892/or.2016.5007

27. Cui Y, Yi L, Zhang MM, et al. WITHDRAWN: knockdown of LncRNA HOXA11 antisense promotes glioma cell apoptosis via sponging MiR-140-5p. Oncol Res. 2017. doi:10.3727/096504017X 14972669062547

28. Tian Y, Nan Y, Han L, et al. MicroRNA miR-451 downregulates the PI3K/AKT pathway through CAB39 in human glioma. Int J Oncol. 2012;40(4):1105-1112.

29. Wei Y, Jiang Y, Zou F, et al. Activation of PI3K/Akt pathway by CD133-p85 interaction promotes tumorigenic capacity of glioma stem cells. Proc Natl Acad Sci U S A. 2013;110(17):6829-6834. doi:10.1073/pnas.1217002110

\section{Publish your work in this journal}

Cancer Management and Research is an international, peer-reviewed open access journal focusing on cancer research and the optimal use of preventative and integrated treatment interventions to achieve improved outcomes, enhanced survival and quality of life for the cancer patient.
The manuscript management system is completely online and includes a very quick and fair peer-review system, which is all easy to use. Visit http://www.dovepress.com/testimonials.php to read real quotes from published authors. 